\title{
Recurrence of pulmonary embolism during anticoagulant treatment: a prospective study
}

\author{
PHILIPPE GIRARD, MARC MATHIEU, GÉRALD SIMONNEAU, PATRICK \\ PETITPRETZ, JACQUES CERRINA, PHILIPPE HERVE, JEAN ROSSO, \\ DOMINIQUE MUSSET, JEANNIE MENSCH, PIERRE DUROUX
}

From the Service de Pneumologie et Réanimation and the Service de Radiologie, Hôpital A Béclère, Clamart, France

ABSTRACT The risk of early recurrence of pulmonary embolism in patients with venous thromboembolic disease treated by anticoagulants is not well established. To determine the risk linked to contemporary proximal deep venous thrombosis, a prospective study was organised to give clinical and scintigraphic surveillance to 50 patients with angiographically proved pulmonary embolism plus phlebographically proved proximal deep vein thrombosis during the first 15 days of anticoagulant treatment. Perfusion lung scans were performed initially and on days 3,7 , and 15 . Only two patients had a recurrence of pulmonary embolism during this period; both episodes were revealed by new symptoms, and one recurrence was fatal. The systematic performance of angiography in four patients found to have new scintigraphic defects led to the the diagnosis of "spurious scintigraphic recurrence" in three of them. It is concluded that (a) adjusted anticoagulant treatment showed an effectiveness of $96 \%$ for preventing early recurrence of pulmonary embolism in this group of supposed high risk patients, and $(b)$ in patients with recent pulmonary embolism new defects on systematic perfusion lung scans are not specific indicators of recurrent pulmonary embolism.

The risk of early recurrence of pulmonary embolism during anticoagulant treatment is not well established: the frequency given in published studies varies from zero to $28 \%$ during the initial course of intravenous heparin - that is, usually, during first seven to 15 days of treatment. ${ }^{1-11}$ This variation probably has several explanations, some of which, such as the method of heparin administration and the "adequacy" of anticoagulation, have been investigated in prospective comparative studies. ${ }^{2-4910}$ Other important factors possibly contributing to these variable results are: (1) only two of these studies $^{911}$ included systematic reassessment by perfusion lung scanning, and these two studies reported the highest recurrence rates $(28 \%$ and $23 \%)$. (2) In all the reported studies the diagnosis of recurring pulmonary embolism was based on clinical or scintigraphic evi-

\footnotetext{
Address for reprint requests: Dr Philippe Girard, Service de Pneumologie et Réanimation, Hôpital A Béclère, 157 rue de la Porte de Trivaux, 92141 Clamart, France.

Accepted 19 January 1987
}

dence, or both, and hence was not definitively established. None used pulmonary angiography as the sole diagnostic endpoint for both pulmonary embolism and subsequent recurrences. (3) Because lower limb phlebography was not systematically performed, patients with associated proximal deep venous thrombosis, who represent a high risk group for pulmonary embolism ${ }^{12}$ and, presumably, for recurrence of pulmonary embolism, could not be assessed as a separate subgroup.

We have therefore prospectively evaluated the risk of recurrence of pulmonary embolism during the first 15 days of anticoagulant treatment in a homogenous group of patients with both pulmonary embolism and proximal deep vein thrombosis. Such patients seemed likely to have an especially high risk of early recurrence of pulmonary embolism. The study included periodic reassessment by perfusion lung scanning, and the diagnosis of thromboembolic disease was based on the two "gold standards" of pulmonary angiography for pulmonary embolism (both in the initial diagnosis and in confirmation of recurrences) and phlebography for deep vein thrombosis. 
Methods

\section{INCLUSION CRITERIA}

From 30 September 1981 to 30 October 1983 all patients admitted to the department of pulmonary diseases were included in the study if the following criteria were met: pulmonary embolism proved by angiography within the past $\mathbf{4 8}$ hours; proximal deep vein thrombosis (popliteal, femoral, or iliac) proved by phlebography within the past 48 hours (patients with evidence of clot affecting the inferior vena cava excluded); no contraindication to heparin treatment; no clinical shock or acute right ventricular failure requiring rapid removal of embolic obstruction by thrombolytic agents or surgical embolectomy ${ }^{13}$; no evidence of previous significant cardiopulmonary disease or of advanced neoplasia; informed consent obtained. Fifty patients met these criteria.

\section{DIAGNOSTIC METHODS}

Phlebography was performed on recumbent patients by bolus injection of isotonic contrast medium (Hexabrix, Guerbet Laboratoires, France) via "scalp" needles inserted in a dorsal vein of each foot. Tourniquets were placed around the lower and upper third of the calf, and around the lower third of the thigh. Films were taken while the tourniquets were being sequentially moved upward from the ankle to the thigh. At least one additional film was taken during a Valsalva manoeuvre or an abdominal compression to obtain better visualisation of the iliac veins and vena cava. The films were anteroposterior views and they allowed analysis of the appearances of calf, popliteal, and femoral deep veins in all patients. In nine out of the 50 patients, further opacification of iliac veins or inferior vena cava or both was needed; this was performed by percutaneous catheterisation of one or both femoral veins at the groin.

The diagnosis of recent thrombus was based on the appearance of a constant intraluminal filling defect within an opacified vein. The visualisation of a thin white line representing contrast medium between the thrombus and the vein wall on each side of the proximal part of a clot defined a "floating" or "tail" clot. Non-opacification of a vein was taken as indicating thrombosis only if the veins above and below and the collaterals were filled with contrast medium.

Pulmonary angiography was performed in eight patients with suspected submassive pulmonary embolism by injecting the contrast medium "with the stream" in an arm vein; this technique confirmed severe pulmonary vascular obstruction in all eight cases and, in these selected patients, appeared reliable. In all the other patients the injection was made in the pulmonary artery, either in the trunk ("global" opacification) or in the right and left pul- monary arteries successively ("selective" opacifica-: tion). Only conventional angiography was used. In $45 \overline{\text { s }}$ of the 50 patients catheterisation was performed byo percutaneous puncture of a basilic vein and femoralo vein catheterisation was used in the others. The scoring system described by Miller et $\mathbf{l}^{14}$ was used tod evaluate the degree of angiographic vascular obstruc-n tion; this gives a score ranging from 0 to 34, with $a_{-}^{\infty}$ maximum of 16 for intraluminal filling defects and $18 \vec{\circ}$ for alterations of peripheral perfusion. In the event of repeat angiography performed because of a suspected ${ }^{\omega}$ recurrence of embolism, the same techniques were used as in the first procedure so that data would be comparable.

The diagnosis of pulmonary embolism depends on the visualisation of one or more intraluminal filling defects or sharp arterial cutoffs in large pulmonaryo arteries. The diagnosis of recurrent pulmonary embolism required (1) the appearance of one or morec intraluminal filling defects or sharp arterial cutoffs in $\overline{<}$ an area not previously affected by the embolic pro- $\overrightarrow{0}$ cess, or (2) more proximal extension of vascularoo obstruction than appeared in the reference angiogram. The disappearance of a proximal clot witho embolisation of more distal arteries in the same area, without any other changes, was interpreted as frag-o mentation of the initial clot, and was not considered $a$ 융 recurrence.

Perfusion lung scanning Microspheres labelled with $\overrightarrow{\overrightarrow{0}}$ technetium-99m albumin were injected intravenously 3 with the patient in the supine position. At least four views were obtained (anterior, posterior, and left and? right lateral). Successive perfusion lung scans were compared only with the immediately preceding one and noted as "better perfusion," "no change," "newx defect or defects," or "normal." One or more new defects suggested recurrence of pulmonary embolism, and therefore led to repeat pulmonary angiography. Interpretation All phlebographic, angiographic, and scintigraphic evidence was interpreted by severals trained chest physicians and radiologists at the time of performance. During the reviewing period at the end of the study all these documents were interpreted N again by two independent pairs of chest physician and radiologist, without knowledge of clinical history or of the relationships between pulmonary angiograms $\omega$ and perfusion lung scans. Only minor differences were found between these two interpretations and between the pairs, and there was no diagnostic change in par- $\bar{\Phi}$ ticular with respect to the diagnosis of recurrent ${ }^{+}$ embolism.

PROTOCOL

Treatment protocol As soon as the diagnosis of venous thromboembolic disease was suspected, a continuous intravenous infusion of sodium heparinates 
was started (500 IU/kg per day, without a loading dose). Further doses were then adjusted on the basis of coagulation tests performed at least once a day for 15 days. The aim was to increase the Howell time to 1.5-2 times the control value, and the thrombin time to more than 2 minutes. In the event of inadequate anticoagulation, the Howell time was rechecked four to eight hours after a therapeutic change. Patients were allowed to become anbulant, using venous elastic support, as soon as satisfactory anticoagulation was achieved.

Surveillance Patients were given daily clinical examinations and perfusion lung scanning was systematically performed on days $0,3,7$ and 15 after entry to the study. The interval between angiography and initial perfusion lung scanning, whatever the order, was less than 36 hours in all patients. Any clinically suspected recurrent pulmonary embolism resulted in a repeat scan and any new defect on perfusion scanning led to repeat pulmonary angiography within 24 hours to confirm embolic recurrence.

\section{Results}

The main clinical data on the 50 patients who met the inclusion criteria during the study period are given in table 1 . The mean (SD) Miller index was 16.1 (5.1)/34 (range 4-27). The location and appearances of proximal venous clots at initial phlebography are presented in table 2 . Deep vein thrombosis was right sided in 23 patients (46\%) left sided in $21(42 \%)$, and bilateral in six patients $(12 \%)$, with bilateral proximal deep vein thrombosis in four patients. Eighteen $(36 \%)$ and nine $(18 \%)$ patients respectively showed no clinical signs suggesting deep vein thrombosis or pulmonary embolism. These latter 27 patients could be recruited for the study because at our institution bilateral lower limb phlebography is routinely performed in patients with pulmonary embolism even in

\section{Table 1 Main clinical data on the 50 patients included in the} study

\begin{tabular}{lc}
\hline Total No of patients & 50 \\
Age (y): mean & $60 \cdot 8$ \\
range & $26-85$ \\
Sex (M:F) & $35: 15$ \\
Underlying condition* & $22(44 \%)$ \\
Previous venous thromboembolism & $2(4 \%)$ \\
Malignant disease & $13(26 \%)$ \\
Recent surgery (< 1 month) & 5 \\
Orthopaedic & 6 \\
Abdominal & 2 \\
Caesarean section & 1 \\
Normal delivery (< 1 month) & 8 \\
Varicose veins & 8 \\
Obesity & 2 \\
Recent long journey & 2 \\
Motor sequelae of stroke & \\
\hline
\end{tabular}

*Patients may have more than one of these conditions.
Table 2 Initial phlebography: upper limit and aspect of proximal venous clots in the 50 patients

\begin{tabular}{lcc}
\hline Location of DVT & No $(\%)^{*}$ & "Floating" \\
\hline Iliac & $15(28)$ & 12 \\
Femoral & $31(57)$ & 23 \\
Popliteal & $8(15)$ & 8 \\
\hline
\end{tabular}

*The total number of deep vein thromboses exceeds 50 because four patients had bilateral proximal thromboses.

DVT-deep vein thrombosis.

the absence of clinical signs of deep vein thrombosis, and patients with proximal deep vein thrombosis without clinical signs of pulmonary embolism undergo systematic "baseline" perfusion lung scanning. These perfusion defects on lung scans were then confirmed by angiography.

Two patients $(4 \%)$ developed a recurrence of pulmonary embolism, revealed in both cases by new clinical symptoms (days 3 and 12). One died despite emergency surgical treatment, which was performed without repeat lung scanning or angiography because of sudden shock and acute cor pulmonale. Anticoagulation had been inadequate over the proceeding 36 hours before recurrence in this patient. Additional details are given in table 3.

The systematic perfusion scanning revealed new segmental perfusion defects in three patients without any new clinical symptoms (day 7 in one case, day 15 in the other two). In all three patients chest radiographs were normal or unchanged. Subsequent angiography (interval under 24 hours) did not confirm the suspected recurrence, leading to the diagnosis of "spurious scintigraphic recurrence." An example is given in the figure. The total of four patients with new scintigraphic defects includes these three patients, and one patient with "true" recurrent pulmonary embolism detailed in table 3.

Table 3 Main clinical data on two men with "true" recurrent pulmonary embolism

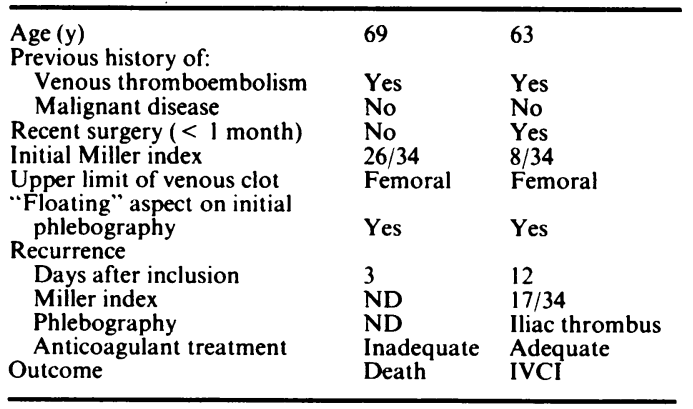

ND—not done; IVCI-inferior vena cava interruption. 


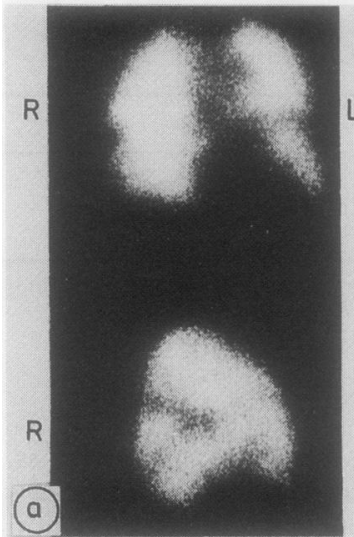

7 th day

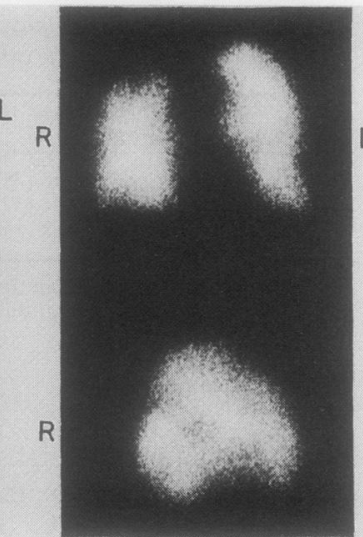

14th day
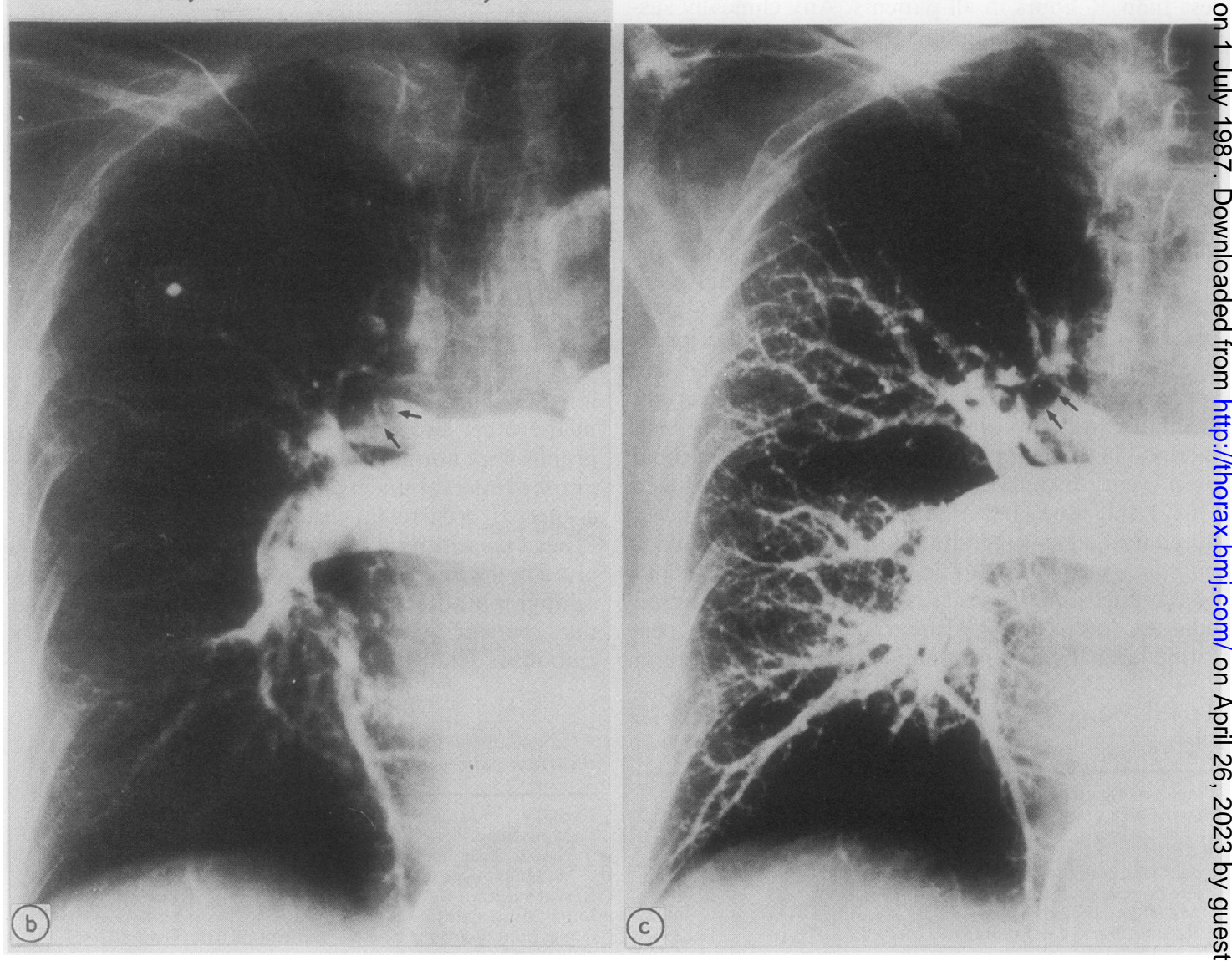

(a) Anterior and right lateral views of systematic perfusion lung scanning performed seven and 14 days after the initial pulmonary angiography. Comparison with the scan obtained seven days after entry into the study shows that an apparently nevo defect had appeared in the right upper lobe, whereas perfusion to the right and left lower lobes had improved. (b) Angiogram (selective right opacification) obtained on the day of entry. There is appreciable hypoperfusion of the right lung with proximal intraluminal filling defects (occlusive and non-occlusive) in all lobar and most segmental arteries. Note the position of the thrombus in the upper lobe pulmonary artery (arrows). (c) Selective right opacification obtained 12 hours after the 14 day perfusion lung scan shown in (a). Comparison with (b) (same times after injection) shows that, although there is a persistent appreciable hypoperfusion of the upper lobe, the intraluminal filling defect is more distal (arrows) and there is a considerable improvement in the perfusion to the middle and lower lobes. 
In three other patients with new clinical symptoms (pleuritic chest pain or haemoptysis or both) repeat lung scans did not show new defects and this was taken to exclude the diagnosis of appreciable recurrence of pulmonary embolism.

Severe complications of heparin treatment occurred in three patients, resulting in the interruption of treatment and the performance of an inferior vena caval interruption procedure. The complications comprised compressive hematomas related to vascular puncture sites in two patients (on day 5 in both cases), and in one patient heparin induced severe thrombocytopenia on day 9 without any clinically detected embolic or haemorrhagic complication.

\section{Discussion}

The risk of pulmonary embolism is especially high in patients with proximal venous thrombosis. ${ }^{12}$ Furthermore, the severity of a recurrence of pulmonary embolism appears to be related both to the size of the recurring embolus and to the residual pulmonary vascular obstruction at the time of recurrence. The first 15 days of treatment correspond to the commonly accepted period for adherence of the thrombus to vein walls ${ }^{15}$ and for the maximum rate of improvement of perfusion as shown by sequential perfusion lung scanning ${ }^{16}$; this therefore represents a period during which the importance and potential severity of recurrent embolism are greatest. Thus by choosing patients with proximal deep vein thrombosis and relatively severe pulmonary embolism (the mean Miller index of 16.8 indicates a mean pulmonary vascular obstruction of $50 \%$ ) we were studying a group of patients with a supposedly high risk of a severe early recurrence of pulmonary embolism.

A recurrence of pulmonary embolism within the first $\mathbf{1 5}$ days of treatment was documented in only two of the 50 patients $(4 \%)$, including one patient whose coagulation test results were inadequate and who died. In the other patient repeat phlebography at the time of recurrence showed proximal extension of the femoral clot despite biologically "adequate" anticoagulation. This latter finding supports the conclusions of Pollak et al, ${ }^{17}$ who suggested that proximal extension of venous clots during anticoagulant treatment was associated with an increased risk of pulmonary embolism. Since repeat phlebography was not performed systematically, however, we could not compare the early evolution of venous clots in patients without a recurrence of pulmonary embolism.

Early caval interruption is sometimes performed routinely in patients with pulmonary embolism plus proximal deep vein thrombosis. ${ }^{1819}$ Our study pro- vides data that might allow the initiation of a prospective randomised comparison of anticoagulation with and without systematic complementary caval interruption. Because of the low incidence of recurrent pulmonary embolism found in the present work, however, such a study would require a very large number of patients: to demonstrate a significant reduction in recurrence rate-for example, from $5 \%$ in the control group treated only by anticoagulation to $1 \%$ in the group with an additional procedureobservations of at least 716 proved cases of acute pulmonary embolism would be necessary. ${ }^{20}$

Systemic performance of pulmonary angiography in four patients with new scintigraphic defects led to the diagnosis of "spurious scintigrâphic recurrence" in three of them (figure 2). This phenomenon, whose frequency is unknown, was first described by Moser $e t$ $a l^{21}$ who attributed it to reperfusion asynchrony between different embolised areas. We must emphasise that our three spurious recurrences, like those described by Moser et al, occurred in very particular conditions: all three patients initially had several embolised areas, the new defects were only segmental, and they occurred in areas where more proximal clots had been seen on the initial angiograms. A retrospective comparison of initial perfusion lung scans with initial angiograms confirmed that areas with normal or subnormal perfusion on scans and nonocclusive thrombi on angiograms could be found in virtually all patients with multiple emboli and a Miller index of over 15. Others have made similar comments on such discrepancies between perfusion lung scans and angiograms. ${ }^{22}$ Thus the present results suggest that, even in patients with recent pulmonary embolism, the specificity of one or more new defects on the perfusion lung scan is insufficient for diagnosing recurrence of pulmonary embolism, especially in the presence of improvement in another area or areas, and indicate that pulmonary angiography should be repeated in such cases to confirm the diagnosis of a recurrence of pulmonary embolism. These results might also help to explain the rather high recurrence rates found in the two studies ${ }^{911}$ that used systematic surveillance by perfusion lung scanning without confirmation of new defects by repeated pulmonary angiography. Finally, we would emphasise that in this study both patients with a true recurrence of pulmonary embolism had very suggestive symptoms, compared with none in the three patients with "spurious recurrences." Thus systematic perfusion lung scanning appears questionable.

Ventilation scanning was not systematically performed in this study. Its usefulness is, however, established only for the initial diagnosis of pulmonary embolism, and it seems improbable that it would have helped to detect "spurious recurrences" because the 
abnormalities giving rise to these appear to be purely vascular. In one patient with "spurious recurrence" in whom a simultaneous ventilation scan was obtained, the "new" perfusion defect was, as expected, "mismatched."

This prospective study, although descriptive and non-comparative, allows two main conclusions. Firstly, adjusted anticoagulant treatment showed an effectiveness of $96 \%$ for preventing early recurrence of pulmonary embolism in our group of patients with pulmonary embolism plus proximal deep vein thrombosis. Secondly, the specificity of perfusion lung scanning for diagnosing early recurrence of pulmonary embolism in such patients is probably insufficient.

\section{References}

1 Barrit DW, Jordan SC. Anticoagulant drugs in the treatment of pulmonary embolism. A controlled trial. Lancet 1960;i:1309-12.

2 Hattersley PG, Mitsuoka JC, King JH. Heparin therapy for thromboembolic disorders. A prospective evaluation of 134 cases monitored by the activated coagulation time. JAMA 1983;250:1413-6.

3 Basu D, Gallus A, Hirsch J, Cade J. A prospective study of the value of monitoring heparin treatment with the activated partial thrombo-plastin time. $N$ Engl $J$ Med 1972;287:324-7.

4 Salzman EW, Deykin D, Shapiro RM, Rosenberg R. Management of heparin therapy. Controlled prospective trial. $N$ Engl J Med 1975;292:1046-50.

5 Coon WW, Willis PW. Recurrence of venous thromboembolism. Surgery 1973;73:823-7.

6 Tibbutt DA, Davies JA, Anderson JA. Comparison by controlled clinical trial of streptokinase and heparin in treatment of life-threatening pulmonary embolism. $\mathrm{Br}$ Med J 1974;i:343-9.

7 Ly B, Arnesen H, Eie H, Hol R. A controlled clinical trial of streptokinase and heparin in the treatment of major pulmonary embolism. Acta Med Scand 1978; 203:465-72.

8 Dotter CT, Seaman AJ, Roesch J. Streptokinase and heparin in the treatment of pulmonary embolism: $a$. randomized comparison. Vasc Surg 1979;13:42-7.

9 Wilson JE, Bynum LJ, Parkey RW. Heparin therapy in venous thromboembolism. Am J Med 1981;70:808-16

10 Glazier RL, Crowell EB. Randomized prospective tria of continuous vs intermittent heparin therapy. JAM 1976;236:1365-7.

11 Urokinase-Pulmonary embolism trial (UPET). Morø bidity and mortality. Circulation 1973;47-48(suppL II):66-72.

12 Moser KM, Lemoine JR. Is embolic risk conditioned by $\overrightarrow{\mathrm{L}}$ location of deep venous thrombosis? Ann Intern Megू 1981;94(part 1):439-44.

13 Petitpretz P, Simonneau G, Cerrina J, et al. Effects of $\underset{3}{\overrightarrow{3}}$ single bolus of urokinase in patients with life threatening pulmonary emboli: a descriptive trial. Cirv culation 1984;70:861-6.

14 Miller GAH, Sutton GC, Kerr IH, Gibson RV, Honey M. Comparison of streptokinase and heparin in treato ment of isolated acute massive pulmonary embolism. Br Med J 1971;ii:681-6.

15 Moser KM. Pulmonary embolism. State of the art. An Rev Respir Dis 1977;115:829-52.

16 Urokinase-pulmonary embolism trial (UPET). Perfusiob lung scanning. Circulation 1973;47-48(suppl II).N 46-50.

17 Pollak EW, De Nardo S, Pollak M, De Nardo G, Wolf mann EF. Detection of anticoagulant therapy failures in patients with venous thrombosis. $\mathrm{Am} J$ Surg 1977;134:214-6.

18 Bernstein EF. The place of venous interruption in the treatment of pulmonary embolism. In: Moser $\mathrm{KM}_{\vec{\sigma}}^{\vec{F}}$ Stern M, eds. Pulmonary thromboembolism. Chicago $\$$ Year Book Publishers, 1973:312-23.

19 Bomalaski JS, Martin GJ, Hughes RL, Yao JST. Infe rior vena cava interruption in the management of pul monary embolism. Chest 1982;82:767-74.

20 Casagrande JT, Pike MG. An improved approximat formula for calculating sample sizes for comparing two binomial distributions. Biometrics 1978;34:483-77

21 Moser KM, Longo AM, Ashburn WL, Guisan M. Spu rious scintiphotographic recurrence of pulmonar $\Phi$ emboli. Am J Med 1973;55:434-43.

22 Urokinase pulmonary embolism trial (UPET). Intere elationships of pulmonary angiograms, lung scans? hemodynamic measurements and fibrinolytic findingso Circulation 1973;47-48(suppl II):73-80. 